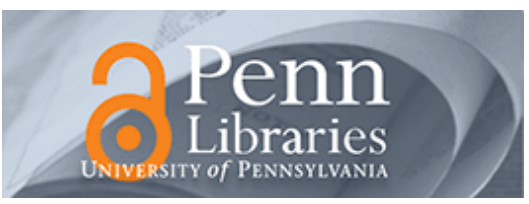

University of Pennsylvania ScholarlyCommons

July 2006

\title{
One-dimensional Diffusion-limited Relaxation of Photoexcitations in Suspensions of Single-walled Carbon Nanotubes
}

\author{
Richard M. Russo \\ University of Pennsylvania \\ Eugene J. Mele \\ University of Pennsylvania, mele@dept.physics.upenn.edu \\ Charles L. Kane \\ University of Pennsylvania, kane@physics.upenn.edu \\ I. V. Rubtsov \\ University of Pennsylvania \\ Michael Therien \\ University of Pennsylvania, therien@a.chem.upenn.edu
}

See next page for additional authors

Follow this and additional works at: https://repository.upenn.edu/mse_papers

\section{Recommended Citation}

Russo, R. M., Mele, E. J., Kane, C. L., Rubtsov, I. V., Therien, M., \& Luzzi, D. E. (2006). One-dimensional Diffusion-limited Relaxation of Photoexcitations in Suspensions of Single-walled Carbon Nanotubes.

Retrieved from https://repository.upenn.edu/mse_papers/112

Postprint version. Published in Physical Review B, Volume 74, Issue 4, Article 041405, July 2006, 4 pages.

Publisher URL: http://dx.doi.org/10.1103/PhysRevB.74.041405

This paper is posted at ScholarlyCommons. https://repository.upenn.edu/mse_papers/112

For more information, please contact repository@pobox.upenn.edu. 


\title{
One-dimensional Diffusion-limited Relaxation of Photoexcitations in Suspensions of Single-walled Carbon Nanotubes
}

\author{
Abstract \\ We report pump-probe transient absorption spectroscopy on carbon nanotubes with a high initial \\ excitation density. We find that the recovery of the ground state optical absorption is well described by a \\ $1 / \sqrt{ } t$ relaxation, indicating that the long time population relaxation is controlled by one-dimensional \\ diffusion limited two body recombination. \\ Comments \\ Postprint version. Published in Physical Review B, Volume 74, Issue 4, Article 041405, July 2006, 4 pages. \\ Publisher URL: http://dx.doi.org/10.1103/PhysRevB.74.041405 \\ Author(s) \\ Richard M. Russo, Eugene J. Mele, Charles L. Kane, I. V. Rubtsov, Michael Therien, and David E. Luzzi
}




\title{
One-dimensional diffusion-limited relaxation of photoexcitations in suspensions of single-walled carbon nanotubes
}

\author{
R. M. Russo, ${ }^{1}$ E. J. Mele,${ }^{2}$ C. L. Kane,${ }^{2}$ I. V. Rubtsov,${ }^{3, *}$ M. J. Therien,${ }^{3}$ and D. E. Luzzi ${ }^{1}$ \\ ${ }^{1}$ Department of Materials Science and Engineering, University of Pennsylvania, Philadelphia, Pennyslvania 19104, USA \\ ${ }^{2}$ Department of Physics, University of Pennsylvania, Philadelphia, Pennyslvania 19104, USA \\ ${ }^{3}$ Department of Chemistry, University of Pennsylvania, Philadelphia, Pennsylvania 19104, USA
}

(Received 2 May 2006; revised manuscript received 29 June 2006; published 26 July 2006)

\begin{abstract}
We report pump-probe transient absorption spectroscopy on carbon nanotubes with a high initial excitation density. We find that the recovery of the ground state optical absorption is well described by a $1 / \sqrt{t}$ relaxation, indicating that the long time population relaxation is controlled by one-dimensional diffusion limited two body recombination.
\end{abstract}

DOI: 10.1103/PhysRevB.74.041405

PACS number(s): 78.67.Ch, 42.50.Md, 73.63.Fg

Single-walled carbon nanotubes (SWNTs) are prototypical one-dimensional (1D) nanomaterials possessing exceptional electronic and mechanical properties. ${ }^{1}$ Recent work emphasizes the interest in SWNTs as optical materials, as well as the potential of unique applications that integrate their electronic and optical characteristics. ${ }^{2,3}$ Both semiconducting and metallic SWNTs absorb light at wavelengths determined by the tube wrapping vector, length, and aggregation state ${ }^{2}$ and fluorescence emission from semiconducting SWNTs has been demonstrated. ${ }^{3}$ Importantly, the fluorescence quantum yield is observed to be extremely low $\left(10^{-3}-10^{-4}\right)$, indicating that most of the excited carriers relax quickly to states that decay by a nonradiative pathway. ${ }^{1,4,5}$ It is unclear whether this relaxation involves defects and/or interaction with external species, or is due to the relaxation of photoexcitations into intrinsic dark excited states.

These observations are stimulating various studies of SWNTs via transient optical spectroscopies using one color and two color pump-probe experiments on surfactant suspensions of SWNTs. ${ }^{6-10}$ Measurements, using these techniques, have led to the development of two models: One treats the system using one-body kinetics, ${ }^{6-8}$ and the other considers a system of interacting excitons using two-body kinetics. ${ }^{9-11}$ The first model proposes a biexponential process for the decay of the bleached ground state absorption spectrum of the $S_{11}$ absorption peak. The fast process is assigned to band edge relaxation, with time constants ranging from 0.3 to 1.2 ps. $^{6-8}$ and the slower process, attributed to interband carrier recombination, has time constants of $5-100$ ps. ${ }^{6-8}$ Other work on thin films of SWNTs, where the tubes are bundled and in intimate contact with each other, recover much more rapidly. ${ }^{12}$ The second model considers excitonic annihilation processes, governed by two-body reaction kinetics, and in some cases ${ }^{9}$ shows excitonic diffusion along the length and circumference of the tube with a decay rate proportional to $1 / t$. In the prior work, the pump fluence ranges from 0.05 to $33 \mathrm{~mJ} / \mathrm{cm}^{2}$, and the temporal range of the experiments was up to $\sim 100 \mathrm{ps}$.

In this paper, we report the results of nanotube transient absorption spectroscopy after pulsed optical excitation at $1.0 \mathrm{~mJ} / \mathrm{cm}^{2}$ with temporal analysis up to $\sim 1 \mathrm{~ns}$. From the methods used in this work, we are able to identify a long time power law decay proportional to $1 / \sqrt{t}$, which persists over four decades in time. This behavior is predicted for diffusion limited pair recombination in one dimension, ${ }^{13,14}$ and is commensurate with the one-dimensional structure of a single-walled carbon nanotube.

The nanotube suspensions were made from material produced by the HipCO process ${ }^{15}$ (batch 81 ) and used without any further purification. The SWNTs were dissolved in a 2.0 wt. \% sodium dodecylbenzene sulfonate (SDBS) solution, and sonicated at high power $(\sim 2.5 \mathrm{~W} / \mathrm{mL})$ with the solution in direct contact with the sonication horn of a cup horn sonicator for $10 \mathrm{~min}$. The surfactant coated, individual SWNTs were separated from the larger SWNT aggregates, and graphitic and catalytic impurities by ultracentrifugation at $122000 \mathrm{~g}$ for $4 \mathrm{~h}$. The upper $60 \%$ of supernatant, which is rich in individual surfactant-coated SWNTs, was collected and used in all further experiments. This procedure is similar to that which is described elsewhere. ${ }^{2}$

The transient absorption spectrum from $920-1430 \mathrm{~nm}$ is obtained by standard pump-probe methods. A detailed description of the experimental setup can be found elsewhere. ${ }^{16}$ Our experimental conditions differ from many previous pump probe studies in that we use a fixed wavelength pump at $725 \mathrm{~nm}$, and probe the entire $S_{11}$ transient spectrum from 920 to $1430 \mathrm{~nm}$ for delay times in the range $0.2-120 \mathrm{ps}$.

The linear absorption and fluorescence $(720 \mathrm{~nm}$ excitation) spectra of the centrifuged SDBS-SWNT suspension are shown in Fig. 1(a). Due to the structural heterogeneity of the nanotubes present in HipCO material, the absorption spectrum in the NIR has many discrete $S_{11}$ absorption transitions reflecting the dependence of the electronic band structure on variations in the SWNT wrapping vector. Fluorescence excitation in the visible spectrum at $720 \mathrm{~nm}$ shows that only a few of the tubes present have an $S_{22}$ transition in resonance with this excitation wavelength (gray curve). Following the thermalization of the photoexcited species by an intraband process to the $S_{11}$ band edge, a small number of these recombine radiatively. Using the assignments of Bachilo et al., ${ }^{3}$ there are only five SWNTs [with wrapping indices $(10,2)$, $(9,4)$, and $(8,6),(8,7)$, and $(11,4)]$ that have $S_{22}$ transitions within $20 \mathrm{~nm}$ of our transient pump wavelength at $725 \mathrm{~nm}$. However, it is clear, from this and other fluorescence spectra ${ }^{3}$ that tubes that are not in resonance with the excitation wavelength can weakly emit. This effect could either be an off- 

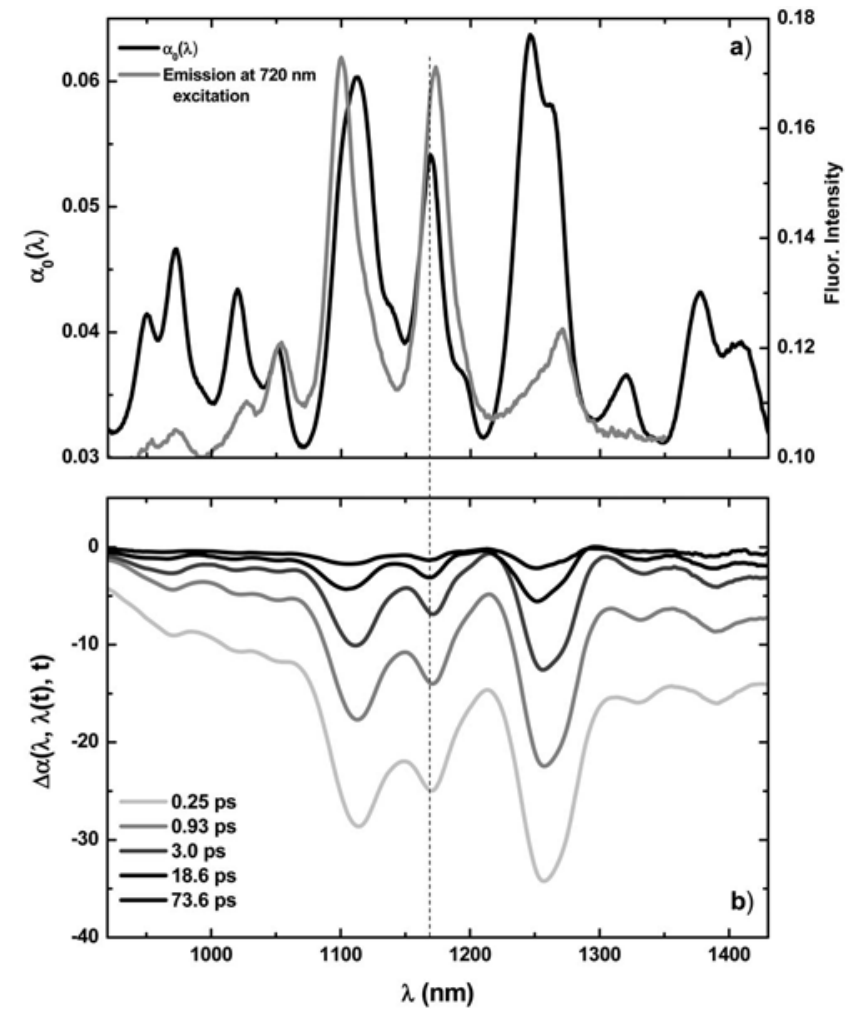

FIG. 1. (a) Linear absorption spectrum (black) and linear fluorescence spectrum (gray) at $720 \mathrm{~nm}$ excitation for $S_{11}$ optical transitions. (b) Transient absorption spectra recorded at several delay times for $\lambda_{\text {pump }}=725 \mathrm{~nm}$ and pump fluence of $\sim 1.0 \mathrm{~mJ} / \mathrm{cm}^{2}$.

resonance excitation into the first or second subband of a nonresonant tube, or be due to emission from small bundles of semiconducting tubes in which at least one is in resonance.

The transient spectrum of the NIR region, using a $725 \mathrm{~nm}$ pump at a fluence of $1.0 \mathrm{~mJ} / \mathrm{cm}^{2}$, is shown in Fig. 1(b). The transient line shape, $\Delta \alpha(\lambda, \bar{\lambda}(t), t)=\alpha(\lambda, \bar{\lambda}(t), t)-\alpha_{0}(\lambda)$, where $\alpha$ is the absorption line shape at time delay $t, \alpha_{0}$ is the ground state absorption line shape, and $\bar{\lambda}(t)$ is the mean wavelength of the absorption line shape at time delay $t$. The ground state absorption is bleached most strongly at 1110, 1170 , and $1250 \mathrm{~nm}$; these features closely resemble the shape and position of the same peaks in the linear absorption spectrum of Fig. 1(a). Our quantitative analysis of this spectrum will focus on the spectral features of the $(8,6)$ tubes because, of the three ground state bleached peaks, the one centered at $1170 \mathrm{~nm}$ contains only contributions from $(8,6)$ and $(12,1)$ tubes. Only the $(8,6)$ tube has an $S_{22}$ transition in resonance with the pump wavelength at $725 \mathrm{~nm}$. The other spectral features, at 1110 and $1250 \mathrm{~nm}$, contain at least four unique SWNT wrapping vectors in each peak. ${ }^{3}$ The bleach transition at $1110 \mathrm{~nm}$ contains contributions from $(9,4)$, $(9,2),(8,4)$, and $(7,6)$ tubes; that at $1250 \mathrm{~nm}$ contains contributions from $(11,1),(10,5),(10,3)$, and $(8,7)$. Note that in the transient spectra of Fig. 1(b) the pump is in resonance with the $S_{22}$ of only a few tubes, however, there are several bleach signatures from nonresonant tubes. We note only that in our analysis of the $(8,6)$ tube, we cannot exclude overlapping
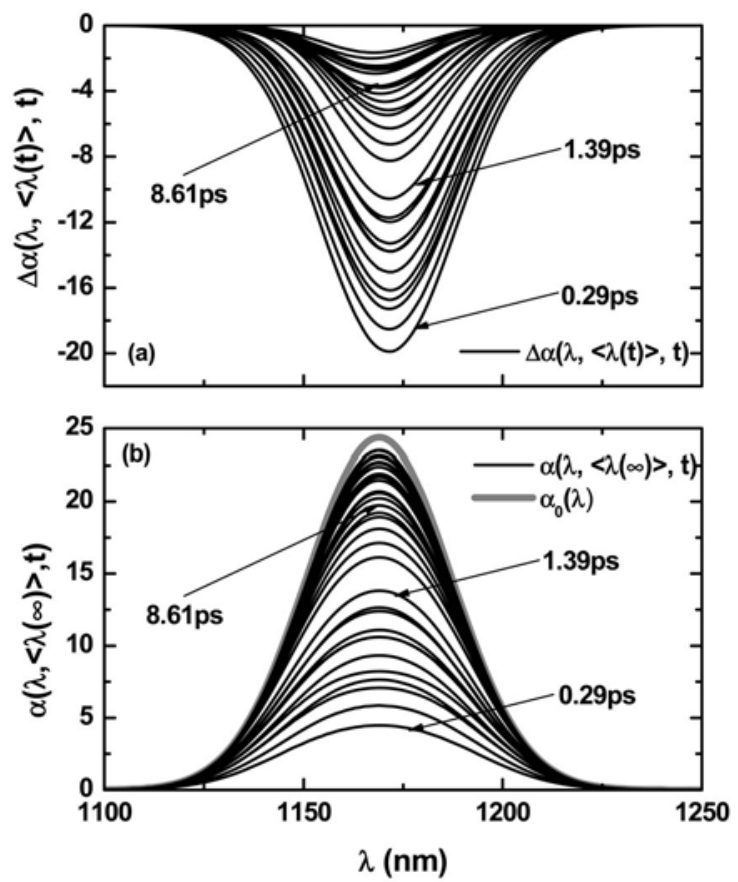

FIG. 2. (a) $\Delta \alpha(\lambda, \bar{\lambda}(t), t)$ for the $(8,6)$ tube, obtained from the deconvolution of the transient spectra over a range of $0.25-113 \mathrm{ps}$. (b) $\alpha(\lambda, \bar{\lambda}(\infty), t)$ for the $(8,6)$ tube, calculated at each time delay using $\alpha(\lambda, \bar{\lambda}(\infty), t)=\Delta \alpha(\lambda, \bar{\lambda}(\infty), t)+\alpha_{0}(\lambda)$.

spectral features from the $(12,1)$ tube, whose $S_{22}$ transition is at $792 \mathrm{~nm}$. Since the excitation density of the pump is sufficient to create a large population of particles on a single tube, and there is some spectral overlap between the $S_{22}$ subbands of both the $(8,6)$ and $(12,1)$ tubes, we expect similar effects in the measured transient absorption for both species.

To evaluate the transient recovery processes of the $(8,6)$ tube, we deconvolute the transient spectra at several time delays ranging from 0.25 to $113 \mathrm{ps}$. This is done using a least squares fitting algorithm with 23 Gaussian line shapes used to represent the 23 distinct SWNT $S_{11}$ absorption peaks that exist over a spectral range of $920-1430 \mathrm{~nm}$, where the mean peak position of the wrapping vector assignments of Bachilo et al. ${ }^{3}$ are used as a starting point for the deconvolution of the transient spectrum. We chose to represent $(8,6)$ and $(12,1)$ as one peak because the difference in peak center positions is $\sim 1 \mathrm{~nm}^{3}$ In our trial fits, when we used two peaks, they were virtually indistinguishable. We repeat the deconvolution process to obtain the Gaussian line shape [Fig. 2(a)], which represents $\Delta \alpha(\lambda, \bar{\lambda}(t), t)$ for our $(8,6)$ tube, for several transient spectra between short $(0.25 \mathrm{ps})$ and long time delays (113 ps).

The $(8,6)$ deconvoluted peak, $\Delta \alpha(\lambda, \bar{\lambda}(t), t)$, is shown in Fig. 2(a). It shows a blue shift in the mean wavelength of bleach as a function of increasing time delay. For all $\Delta \alpha(\lambda, \bar{\lambda}(t), t)$ measured, the mean deviation $|\bar{\lambda}(t)-\bar{\lambda}(\infty)|$, where $\bar{\lambda}(\infty)$ is the mean wavelength of the ground state absorption line shape, is $1.73 \mathrm{~nm}$ with a standard deviation of $0.96 \mathrm{~nm}$. This deviation is small relative to the mean FWHM of the $(8,6)$ tube, which is $29.0 \mathrm{~nm}$ with a standard deviation 

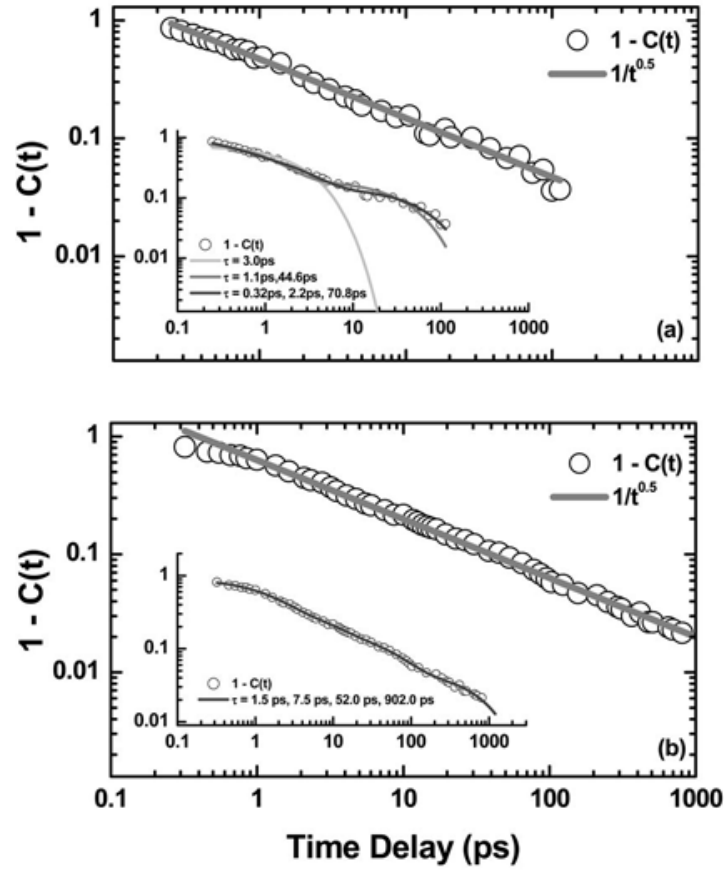

FIG. 3. (a) 1-C(t) (open circles) versus t on a log-log scale for an $(8,6)$ tube. $1-C(t)$ is linear over three decades in time and shows $1 / \sqrt{t}$ dependence. $y=1 / \sqrt{t}$ (line) is shown for comparison. (inset) 1- $C(t)$ compared to mono-, bi-, and tri-exponential fits. (b) Similar linear behavior is observed for a $(7,6)$. (inset) A good fit now requires at least four exponentials.

of $3.0 \mathrm{~nm}$. We correct this deviation by shifting the mean wavelength of the line shape to coincide with that of $\alpha_{0}$. The corrected $\Delta \alpha$ is now written as $\Delta \alpha(\lambda, \bar{\lambda}(\infty), t) . \alpha(\lambda, \bar{\lambda}(\infty), t)$, which is the absorption line shape of the $S_{11}$ subband at time delay $t$, can now be computed using the relation: $\alpha(\lambda, \bar{\lambda}(\infty), t)=\Delta \alpha(\lambda, \bar{\lambda}(\infty), t)+\alpha_{0}(\lambda) . \alpha$ is shown in Fig. 2(b).

We compute the rate of recovery of $\alpha(\lambda, \bar{\lambda}(\infty), t)$ using the relation: $\alpha(\lambda, \bar{\lambda}(\infty), t)=C(t) \alpha_{0}(\lambda)$, where $C(t)$ is zero when the ground state of the tube is completely bleached, and 1 , when it has completely recovered. $C(t)$ represents the portion of the magnitude of ground state absorption recovered at time delay $t$, which we determine for each $\alpha(\lambda, \bar{\lambda}(\infty), t)$. We want to study the remaining excited state population, 1 $-C(t)$. When plotted on a log-log scale [Fig. 3(a)], $1-C(t)$ is linear over three decades in time, and is well described by $y=1 / \sqrt{t}$. A mean field treatment of two-body reaction kinetics predicts a population relaxation of the excitation density $\dot{n}=-\gamma n^{2}$ and thus $n(t) \propto 1 / t$ at long times. However, the mean-field theory fails for diffusion limited pair reactions in one dimension. ${ }^{13,14}$ In one dimension, and assuming that two-body collisions eliminate pairs of excitations with unit probability, one obtains instead the scaling relation $n(t)$ $\propto 1 / \sqrt{t}$ reflecting the diffusion range of a photoexcited species in time $t$. This is consistent with the data in Fig. 3(a) and suggests that 1D diffusion of the long-lived photoexcited state limits the population relaxation for this system.

For comparison, we have fit $1-C(t)$ to mono-, bi-, and tri-exponential decay functions [Fig. 3(a) (inset)]. The mono-, and bi-exponential functions do not give an accurate fit to the experimental data. The triexponential function does, however, fit $1-C(t)$ very well. Examination of the time constants in this fit reveals a spacing of approximately one order of magnitude between each. While it is clear that several exponential functions can be used to fit $1-C(t)$, based on the uniform spacing in the magnitude of the time constants, it seems unlikely that a fit to a sum of exponential functions can have a physical interpretation. However, the electronic transport in SWNTs along their length is well established, and $1-C(t)$, which shows $1 / \sqrt{t}$ dependence, is consistent with a scaling factor for diffusion limited recombination in one dimension.

In Fig. 3(b), 1-C(t) is shown over four decades in time, up to $1000 \mathrm{ps}$, and shows $1 / \sqrt{t}$ dependence over the entire length of the decay. These data have been obtained from a $(7,6)$ tube, where the nanotubes were dissolved in a 2.0 wt. \% surfactant solution using a proprietary amphiphilic oligomer as the surfactant molecule. The suspension was centrifuged at $75000 \mathrm{~g}$ for $4 \mathrm{~h}$, and the upper $60 \%$ of the supernatant was collected for analysis by transient spectroscopy. The transient spectrum was measured using a fluence of $1.0 \mathrm{~mJ} / \mathrm{cm}^{2}$, and a pump wavelength of $670 \mathrm{~nm}$ that coincided with the $S_{22}$ subband of the $(7,6)$ tube.

From the experiments of Islam et al. ${ }^{17}$ the orientationally averaged absorption cross section for a second subband transition is approximately $2.0 \times 10^{5} \mathrm{~cm}^{2} /$ mole of carbon or $\sigma$ $=2 \times 10^{5} \mathrm{~cm}^{2} 8 \pi R L /\left(N_{A} \sqrt{3} a^{2}\right)$ for a tube of radius $R$ and length $L$, where $N_{A}$ is Avogadro's number and $a$ is the graphene lattice constant. In our experiments the excitation fluence, $u_{i}$, is $1.0 \mathrm{~mJ} / \mathrm{cm}^{2}$. Since the temporal width of the excitation pulse is small compared to a typical interband recombination time at low fluence, ${ }^{6-8}$ the pump produces an initial excitation density per unit length of the tube, $n_{i}$ $=(\sigma / L)\left\{u_{i} /[\hbar \omega(R)]\right\}$, where $\omega(R)$ is the frequency of the exciting radiation; thus $n_{i}$ is $0.27 \mathrm{~nm}^{-1}$. This corresponds to an initial state containing approximately 270 excitons per micron length of tube. Importantly, the same power law decay of the photobleaching is observed for several excitation fluences, where $u_{i}$ is in the range $100 \mathrm{~mJ} / \mathrm{cm}^{2}<u_{i}$ $<7.0 \mathrm{~mJ} / \mathrm{cm}^{2}$. Note that these excitation densities are comparable to those previously used to study nonlinear absorption and nonlinear exciton-exciton interactions in nanotubes. ${ }^{9}$ They are three orders of magnitude larger than those used in experiments that have studied the transient decay of the fluorescence in the linear absorption regime. ${ }^{11}$

The $1 / \sqrt{t}$ power law decay, observed for $1-C(t)$, is the signature of diffusion limited two-body recombination in a one-dimensional system. This indicates the formation of a nonemissive but mobile (diffusive) long-lived state following photoexcitation. Likely assignments for this state are a triplet exciton produced by intersystem crossing during the hot carrier relaxation, or a dark singlet exciton produced by intervalley scattering of excitons between the $K$ and $K^{\prime}$ points of the Brillouin zone. ${ }^{18-20}$

At a sufficiently long time, the excited tube will evolve to its dilute regime with a relaxation rate that should be limited by the slowest one-body relaxation kinetics. The fluorescence transient beyond this crossover time has been studied 
recently by Hagen et al. that suggest thermal detrapping limits the long time relaxation. ${ }^{21}$ Note also that for an exciton diffusion constant $D=100 \mathrm{~cm}^{2} / \mathrm{s}$ (Ref. 22) an exciton can diffuse the length of a $1 \mu \mathrm{m}$ tube in approximately $100 \mathrm{ps}$. In our samples, the $1 / \sqrt{t}$ recovery persists well beyond this crossover time. However, this crossover time will be sample dependent, and nanotubes are known to have significant variation even if produced by seemingly equivalent synthetic processes. $^{23}$

In conclusion, we report on an example of a 1D diffusion limited recombination process in SWNTs that shows $1 / \sqrt{t}$ dependence. This strongly suggests pairwise recombination as an intrinsic relaxation process for photoexcitations created at high density on a SWNT.

This work was supported in part by the Commonwealth of Pennsylvania's Ben Franklin Technology Development Authority through the Ben Franklin Technology Partners of Southeastern Pennsylvania and the Nanotechnology Institute. This work was supported through the MRSEC Program of the National Science Foundation (DMR-00-79909), the Department of Energy (FG02-ER45118) (E.J.M. and C.L.K.) and the Office of Naval Research (N00014-97-0317) (M.J.T.).
*Current address: Department of Chemistry, Tulane University, New Orleans, LA 70118.

${ }^{1}$ Carbon Nanotubes: Synthesis, Structure, Properties and Applications, edited by M. S. Dresselhaus, G. Dresselhaus, and Ph. Avouris (Springer, New York, 2001).

${ }^{2}$ M. J. O'Connell et al., Science 297, 593 (2002).

${ }^{3}$ S. M. Bachilo et al., Science 298, 2361 (2002).

${ }^{4}$ S. Lebedkin et al., J. Phys. Chem. B 107, 1949 (2003).

${ }^{5}$ M. Jones et al., Phys. Rev. B 71, 115426 (2005).

${ }^{6}$ G. N. Ostojic et al., Phys. Rev. Lett. 92, 117402 (2004).

${ }^{7}$ J. Kono et al., Appl. Phys. A 78, 1093 (2004).

${ }^{8}$ L. Huang, H. N. Pedrosa, and T. D. Krauss, Phys. Rev. Lett. 93, 017403 (2004)

${ }^{9}$ Y. Z. Ma et al., J. Chem. Phys. 120, 3368 (2004).

${ }^{10}$ Y. Z. Ma et al., Phys. Rev. Lett. 94, 157402 (2005).

${ }^{11}$ F. Wang, G. Dukovic, L. E. Brus, and T. F. Heinz, Phys. Rev. Lett.
92, 177401 (2004).

${ }^{12}$ J. S. Lauret et al., Phys. Rev. Lett. 90, 057404 (2003).

${ }^{13}$ D. C. Torney and H. M. McConnell, J. Phys. Chem. 87, 1941 (1983).

${ }^{14}$ D. Toussaint and F. Wilczek, J. Chem. Phys. 78, 2642 (1983).

${ }^{15}$ P. Nikolaev et al., Chem. Phys. Lett. 313, 91 (1999).

${ }^{16}$ I. V. Rubtsov et al., J. Am. Chem. Soc. 125, 2687 (2003).

${ }^{17}$ M. F. Islam et al., Phys. Rev. Lett. 94, 019901(E) (2005).

${ }^{18}$ H. Zhao and S. Mazumdar, Phys. Rev. Lett. 93, 157402 (2004).

${ }^{19}$ V. Perebeinos, J. Tersoff, and Ph. Avouris, Phys. Rev. Lett. 92, 257402 (2004)

${ }^{20}$ C. D. Spataru et al., Phys. Rev. Lett. 92, 077402 (2004).

${ }^{21}$ A. Hagen et al., Phys. Rev. Lett. 95, 197401 (2005).

${ }^{22}$ O. J. Korovyanko et al., Phys. Rev. Lett. 92, 017403 (2004).

${ }^{23}$ W. Zhou et al., Chem. Phys. Lett. 350, 6 (2001). 